https://helda.helsinki.fi

\title{
Optical and magnetic properties of antiaromatic porphyrinoids
}

\section{Valiev, Rashid R.}

2017-10-14

Valiev , R R , Fliegl , H \& Sundholm , D 2017 , ' Optical and magnetic properties of antiaromatic porphyrinoids ', Physical Chemistry Chemical Physics, vol. 19 , no. 38 , pp. 25979-25988 . https://doi.org/10.1039/c7cp05460b

http://hdl.handle.net/10138/309500

https://doi.org/10.1039/c7cp05460b

unspecified

acceptedVersion

Downloaded from Helda, University of Helsinki institutional repository.

This is an electronic reprint of the original article.

This reprint may differ from the original in pagination and typographic detail.

Please cite the original version. 


\title{
Optical and magnetic properties of antiaromatic porphyrinoids
}

\author{
Rashid R. Valiev ${ }^{* a, c}$, Heike Fliegl, ${ }^{* b}$ Dage Sundholm, ${ }^{* c \neq}$
}

Received Xth $X X X X X X X X X X 20 X X$, Accepted Xth $X X X X X X X X X 20 X X$

First published on the web Xth $X X X X X X X X X X 200 X$

\section{DOI: $10.1039 / \mathrm{b} 000000 x$}

Magnetic and spectroscopic properties of a number of formally antiaromatic carbaporphyrins, carbathiaporphyrins and isophlorins with $4 n \pi$ electrons have been investigated at density functional theory and $a b$ initio levels of theory. The calculations show that the paratropic contribution to the magnetically induced ring-current strength susceptibility and the magnetic dipole-transition moment between the ground and the lowest excited state are related. The vertical excitation energy (VEE) of the first excited state decreases with increasing ring-current strength susceptibility, whereas the VEE of the studied higher-lying excited states are almost independent of the size of the ring-current strength susceptibility. Strong antiaromatic porphyrinoids, based on the magnitude of the paratropic ring-current strength susceptibility, have small energy gaps between the highest occupied and lowest unoccupied molecular orbitals and a small VEE of the first excited state. The calculations show that only the lowest $S_{0} \rightarrow S_{1}$ transition contributes signficantly to the magnetically induced ring-current strength susceptibility of the antiaromatic porphyrinoids. The decreasing optical gap combined with a large angular momentum contribution to the magnetic transition moment from the first excited state explains why molecules III-VII are antiaromatic with very strong paratropic ring-current strength susceptibilities. The $\mathrm{S}_{0} \rightarrow S_{1}$ transition is a magnetic dipole-allowed electronic transition that is typical for antiaromatic porphyrinoids with $4 n \pi$ electrons.

\section{Introduction}

Porphyrins are macroheterocycles with four pyrrole rings connected by methine bridges. ${ }^{1}$ Classic porphyrins such as free-base porphyrin, tetraphenylporphyrin and tetrabenzoporphyrin are aromatic molecules satisfying Hückel's $(4 n+2) \pi$-electron count rule. ${ }^{2-4}$ Aromaticity and aromatic pathways of classic porphyrins have been computationally studied by several research groups. ${ }^{5-18}$ Presently, the most reliable approach for assigning the aromatic character of molecules according to the magnetic criterion is by investigating magnetically induced current densities. ${ }^{13,19-22}$ Classic porphyrins have an almost equal degree of aromaticity. However, they show significant differences in the aromatic pathways, ${ }^{13,23}$ while the degree of aromaticity of porphyrinoids like carbaporphyrins and carbathiaporphyrins differs from that of the classic porphyrins. ${ }^{24-29}$ Porphyrinoids play an important role in catalysis and have an ability to form complexes with metals having unusual oxidation states. ${ }^{24,26,30-33}$ Air stable antiaromatic porphyrinoids such as isophlorins have also been synthesized. ${ }^{34-36}$ Antiaromatic air and water stable porphyrinoids such as $\mathrm{Ni}$ (II)norcorrole has recently received attention, since it has been shown that $\mathrm{Ni}$ (II)-norcorrole is suitable as a cathode-active material for battery applications. ${ }^{37}$ Syntheses of expanded porphyrins ${ }^{38-40}$ and contracted porphyrins ${ }^{41,42}$ with aromatic and antiaromatic character have also been reported.

Beside their varying aromatic characteristics, porphyrinoids also show interesting spectroscopic properties that link them to possible applications as dyes in dye-sensitized solar cells, photosensitzers in photodynamic cancer therapy as well as in molecular electronics. ${ }^{43-48}$ Due to their high applicability in various fields, it is desirable to un- derstand the electronic structure of porphyrinoids in detail, which may open up routes to a tailored design of new porphyrinoid based molecules with desired properties. ${ }^{49,50}$ Common spectroscopic techniques that have been used for investigating porphyrinoids are nuclear magnetic resonance (NMR), ${ }^{51}$ ultraviolet (UV) absorption, ${ }^{1}$ magnetic circular dichroism (MCD), ${ }^{52,53}$ electronic circular dichroism (ECD), ${ }^{54,55}$ photoelectron (PE), ${ }^{56}$ and two-photon absorption (TPA) ${ }^{57-59}$ spectroscopies to mention the most common ones.

The absorption spectrum of traditional porphyrins consists of weak $Q_{x}$ and $Q_{y}$ bands that appear in the red part of the visible region. ${ }^{60}$ Additional peaks appearing in the $Q$-band region of the absorption spectrum are due to vibronic progression of $\mathrm{S}_{0} \rightarrow S_{1}$ and $\mathrm{S}_{0} \rightarrow \mathrm{S}_{2}$ electronic transitions, ${ }^{61}$ while the strong Soret $B_{x}$ and $B_{y}$ bands are usually broad peaks in the violet region without any fine structure. ${ }^{1,60,62}$ The $S_{0} \rightarrow S_{1}-S_{4}$ transitions of the classic porphyrins can be understood by employing Gouterman's fourorbitals model, ${ }^{60}$ whereas there is no detailed theoretical analysis of the electronic transitions of aromatic carbaporphyrins and carbathiaporphyrins. ${ }^{63}$ However, it is generally assumed that their electronic transitions are similar to those of the classic porphyrins due to their similar structure and aromatic nature. Experimentally, the electronic absorption spectra of carbaporphyrins and carbathiaporphyrins also show weak (dark) $Q_{x}$ and $Q_{y}$ bands in the lowenergy part of the visible spectrum and a strong Soret band $\left(B_{x}, B_{y}\right)$ in the near ultraviolet region. ${ }^{24-26}$ Computational studies of the spectroscopic properties of antiaromatic carbaporphyrins, carbathiaporphyrins and isophlorins might reveal some interesting features in the absorption spectra that are not easily obtained spectroscopically. 
We previously carried out an analysis of the magnetic properties and electronic excitation spectra of strongly antiaromatic isophlorins, ${ }^{64}$ whereas in this work we investigate transitions between the ground state and excited electronic states of seven formally antiaromatic porphyrinoids with $4 n \pi$ electrons. The aim of the study is to elucidate whether there is a relation between the antiaromatic character and absorption spectra. For closed-shell molecules, the magnetic dipole-allowed transitions between singlet states are completely determined by the off-diagonal matrix elements over the angular momentum operator. The paramagnetic contribution to magnetically induced current densities can be expressed using the same matrix elements. ${ }^{22,65,66}$ Thus, a correlation between the two apparently different properties cannot be ruled out.

The paper is organized in the following manner. The computational details are given in Section 2 and some theoretical relations are presented in Section 3. The calculated ring-current strength susceptibilities and spectroscopic properties are discussed in Section 4. The final conclusions are drawn in Section 5.

\section{Computational methods}

The ground-state molecular structures of the studied molecules have previously been optimized at the density functional theory (DFT) level. ${ }^{27,28,64}$ The molecular structure optimizations were performed using Becke's threeparameter functional in combination with the Lee-YangParr correlation functional (B3LYP). ${ }^{67,68}$ The Karlsruhe def2-TZVP basis sets have been employed in all calculations. ${ }^{69,70}$ The prefix def 2 is omitted in the discussion. The reported ring-current strength susceptibilities have previously been calculated at the B3LYP/def2-TZVP level. ${ }^{27,28,64}$ The ring-current strength susceptibilities were obtained by integrating the current density flow that passes through a plane placed perpendicularly to the porphyrinoid macroring. The external magnetic field was also directed perpendicularly to the macroring. The optimized molecular structures and the ring-current strength susceptibilities calculated at the B3LYP level have been taken from our previous works. ${ }^{27,28,64}$ The ring-current strength susceptibilities calculated at the Hartree-Fock (HF/def2-TZVP) and secondorder Møller-Plesset perturbation theory (MP2/def2-TZVP) levels have been taken from Ref. 71.

Vertical excitation energies (VEE), electric dipole and magnetic dipole-allowed transition moments for the $S_{0} \rightarrow S_{n}$ transitions with $n=1-5$ have been calculated at the timedependent density functional theory (TDDFT) level with

$\dagger$ Electronic Supplementary Information (ESI) available: The HOMOLUMO transitions, individual transition moments as well as frontier molecular orbital plots for the molecules investigated are reported. See DOI: 10.1039/b000000x/

${ }^{a}$ Tomsk State University, Lenina 36, Tomsk, Russian Federation. E-mail: valievrashid@gmail.com,

${ }^{b}$ Centre for Theoretical and Computational Chemistry (CTCC), Department of Chemistry, University of Oslo, P.O.Box 1033 Blindern, 0315 Oslo, Norway, E-mail: heike.fliegl@kjemi.uio.no

${ }^{c}$ Department of Chemistry, P.O. Box 55 (A.I. Virtanens plats 1), FIN-00014 University of Helsinki, Finland, E-mail: dage.sundholm@helsinki.fi the B3LYP functional and the def2-TZVP basis set using Gaussian 09 version A.02. ${ }^{72,73}$

The VEEs have also been calculated at the approximate second-order coupled-cluster level (CC2) using the def2TZVP basis sets and the resolution of identify (RI) approximation as implemented in Turbomole. ${ }^{74-76}$ The reducedvirtual-space (RVS) approach with an energy threshold of $50 \mathrm{eV}$ was used in the CC2 calculations. ${ }^{77}$ The RVS approach leads to a significant reduction of the computational time rendering CC2 calculations on large molecules feasible. ${ }^{78}$ The RVS approximation yields VEEs at $a b$ initio correlation levels that are in close agreement with those obtained using the full virtual space. ${ }^{79,80}$

\section{Theory}

Magnetically induced current densities can be formally expressed as a sum of diamagnetic and paramagnetic contributions ${ }^{22,66,81}$

$$
\begin{aligned}
J & =J^{d}+J^{p} \\
& =-\left(\frac{e^{2}}{m_{e}}\right) A \psi_{0}^{2}-i\left(\frac{e \hbar}{2 m_{e}}\right) \sum_{n \neq 0}\left(c_{n}-c_{n}^{*}\right)\left(\psi_{n} \nabla \psi_{0}-\psi_{0} \nabla \psi_{n}\right)
\end{aligned}
$$

where $J^{d}$ and $J^{p}$ are the diamagnetic and paramagnetic parts of the current density. $A$ is the vector potential of the external magnetic field, $e$ is the charge of the electron, and $m_{e}$ is its mass. $\psi_{0}$ is the unperturbed ground-state wave function and $\psi_{n}$ are unperturbed wave functions of the excited states. The division into diamagnetic and paramagnetic contributions is gauge-origin dependent. The expansion coefficients of the first-order perturbed wave function in the basis of the unperturbed excited states $\left(c_{n}\right)$ are given by

$$
c_{n}=\frac{\langle n|\hat{L}| 0\rangle}{\Delta E_{n 0}} .
$$

$\Delta E_{n 0}$ is the electronic excitation energy from the ground state to the $n$-th excited state. $\hat{L}$ is the angular momentum operator, which can be replaced by $\hat{L}_{z}$ when considering external magnetic field along the $z$ axis that is perpendicular to the porphyrinoid macroring.

The matrix elements $\langle n|\hat{L}| 0\rangle$ are proportional to the paramagnetic contribution of the current density, whereas the diamagnetic contribution can be obtained from the groundstate wave function.

The transition moment of a magnetic dipole-allowed transition between the initial $|0\rangle$ and final $|n\rangle$ state is given by the transition matrix element over the magnetic dipole operator $\hat{M}$

$$
M_{n 0}=\langle n|\hat{M}| 0\rangle \text { with } \hat{M}=\mu_{B}\left(\hat{L}+g_{e} \hat{S}\right)
$$

where $\mu_{B}$ is the Bohr magneton, $g_{e}$ is the electronic $g$ factor, $\hat{L}$ is the angular momentum operator and $\hat{S}$ is the electronic spin operator. Since we consider only closedshell molecules, the matrix elements over $\hat{S}$ vanish and only matrix elements containing the angular momentum 
operator $L_{n 0}=\langle n|\hat{L}| 0\rangle$ contribute to the magnetic dipoleallowed transition $\mathrm{S}_{0} \rightarrow S_{n}$. The matrix elements involving the $z$ component of the angular momentum operator $\left(\hat{L}_{z}\right)$, which originate from the circular electron motion around the molecular ring, are the largest contributions to the magnetic dipole-allowed transition moments.

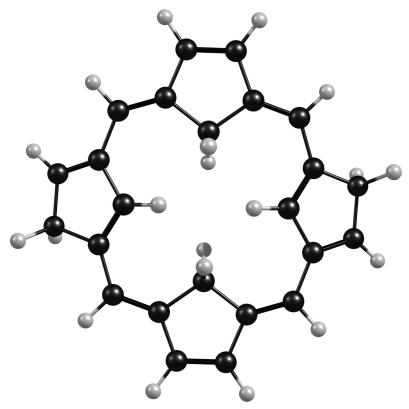

(a) (I)

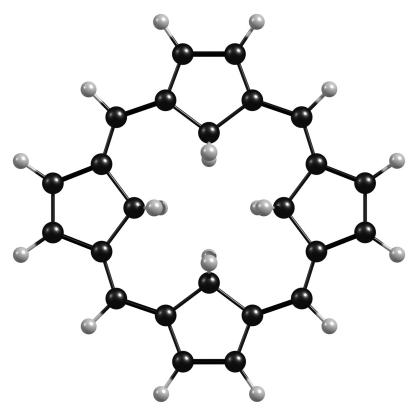

(c) (III)

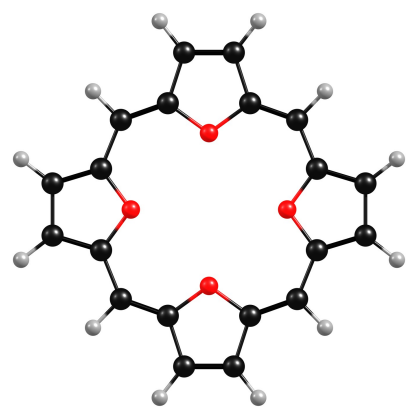

(e) (V)

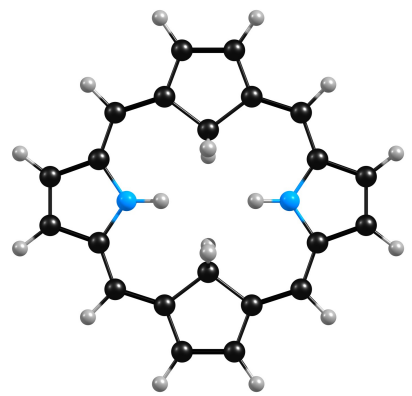

(g) (VII)

Fig. 1 The molecular structures of the investigated porphyrinoids.

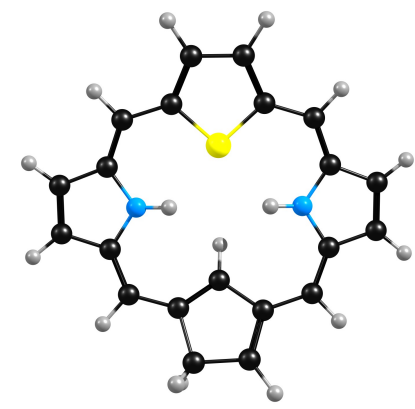

(b) (II)

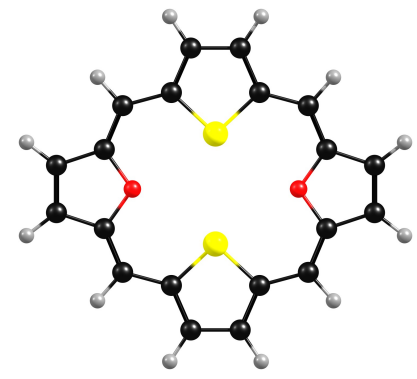

(d) (IV)

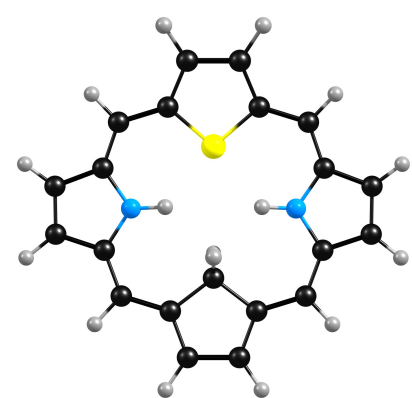

(f) (VI)

\section{Results and discussion}

\section{Molecular structures}

Molecule I is a 2,12,22,24-H-tetracarbaporphyrin, where the four $\mathrm{NH}$ moieties of isophlorin ${ }^{82}$ are replaced with $\mathrm{CH}_{2}$ and two of the five-member rings are inverted (confused). Molecule II is a carbaporphyrin that is obtained by replacing one of the $\mathrm{NH}$ units of isophlorin with $\mathrm{S}$ and the $\mathrm{NH}$ unit in the trans position with respect to $S$ is replaced with $\mathrm{CH}_{2}$ and the ring is inverted. Molecule III is $21,22,23,24-$ $\mathrm{H}$-tetracarbaporphyrin, where all $\mathrm{NH}$ moieties of isophlorin have been replaced by $\mathrm{CH}_{2}$.

The IV and V molecules are dioxa-dithiaisophlorin and tetraoxa-isophlorin. The synthesized compounds IV and $\mathrm{V}$ have fluorinated phenyl groups in the meta-positions. ${ }^{34}$ However, since the substituents do not significantly influence the spectroscopic and aromatic properties, they are replaced with hydrogens to reduce the computational costs.

In molecule VI, one of the $\mathrm{NH}$ units of isophlorin is replaced with $S$ and the one in the trans position with respect the $\mathrm{S}$ is replaced with an isoelectronic $\mathrm{CH}_{2}$ moiety. Molecule VII is 21,22,23,24-H-21,23-dicarbaporphyrin with two of the inner $\mathrm{NH}$ moieties of isophlorin replaced with $\mathrm{CH}_{2}$ moieties.

Carbaporphyrins I, III and VII have not been synthesized, while carbaporphyrins II and VI as well as isophlorins IV and $\mathrm{V}$ have been synthesized and computationally characterized. $24,27,28,34,64$ The molecular structures of the studied antiaromatic porphyrinoids are shown in Figure 1.

Table 1 The ring-current strength susceptibilities (in $n A / T$ ) calculated at the Hartree-Fock (HF), density functional theory (DFT) and second-order Møller-Plesset perturbation theory (MP2) levels of theory. The B3LYP functional was used in the DFT calculations.

\begin{tabular}{lccc}
\hline \hline Molecule & HF & DFT & MP2 \\
\hline I & -0.3 & -1.1 & -0.6 \\
II & -2.4 & -7.4 & -4.2 \\
III & -8.6 & -35.0 & -17.5 \\
IV & -13.9 & -52.5 & -28.7 \\
V & -15.9 & -62.5 & -27.4 \\
VI & -18.5 & -76.8 & -34.9 \\
VII & -20.7 & -145.0 & -48.6 \\
\hline \hline
\end{tabular}

\section{Ring-current strength susceptibilities}

The aromatic character of the investigated porphyrinoids can be understood by considering that the planar molecules have practically circular symmetry whose oneparticle functions have an angular dependence of $\exp (\operatorname{im} \varphi)$, where $m$ is the azimuthal quantum number for rotation $\varphi$ around the symmetry axis at the center of the porphyrinoid ring. In this representation, the two first valence $\pi$ electrons can be considered to occupy the total symmetric function corresponding to $m=0$, whereas the $\pm m$ functions with $m \geq 1$ are degenerate hosting four electrons. 
Aromatic porphyrinoids with $4 n+2 \pi$ electrons are formally closed-shell molecules in this approximate representation, whereas antiaromatic porphyrinoids with $4 n \pi$ electrons have formally only two electrons in the outermost $m$ shell that can couple to a singlet or a triplet. However, the present calculations show that the ground state of the studied antiaromatic porphyrinoids are closed-shell singlet states with a significant optical gap.

The magnetically induced ring-current strength susceptibilities are a very reliable means for assessing the (anti)aromatic character of molecules according to the magnetic criterion. ${ }^{65,83}$ Diatropic and paratropic current densities flow in opposite directions around the porphyrinoid macroring when the molecule is exposed to an external magnetic field in the $z$ direction i.e., perpendicularly to the molecular ring. Diatropic currents are assumed to circle in the classical direction (clockwise). Aromatic molecules are dominated by diatropic ring currents, while antiaromatic molecules sustain net paratropic ring currents. Nonaromatic molecules also sustain magnetically induced currents. However, the strengths of the diatropic and paratropic ring currents are then of the same size and cancel. ${ }^{22,83,84}$ Cyclobutadiene, which is the archetypal antiaromatic molecule, sustains a net current strength susceptibility of $-19.9 \mathrm{nA} / \mathrm{T}$ at the B3LYP/TZVP level of theory, which can be compared with the ring-current strength susceptibility of $12.0 \mathrm{nA} / \mathrm{T}$ for benzene calculated at the same level. Ring-current strength susceptibilities are obtained by integrating the current-density flow passing selected bond(s) of the investigated molecular ring. ${ }^{22}$

The molecules are ordered such that the degree of antiaromaticity judged from the ring-current strength criterion obtained at the B3LYP level increases with molecule number. The ring-current strength susceptibilities calculated at the HF, B3LYP and MP2 levels of theory are given in Table 1 . The ring-current strength susceptibilities calculated at the HF level are much smaller than the B3LYP ones. For the almost nonaromatic molecules I and II, the HF and MP2 calculations yield practically the same ringcurrent strength susceptibilities, whereas for the strongly antiaromatic porphyrinoids, the ring-current strength susceptibilities calculated at the MP2 level are about a factor of two larger than the HF ones. B3LYP calculations overestimate the ring-current strength susceptibility by a factor of two as compared to the values obtained at the MP2 level, because the first excitation energy is underestimated at the B3LYP level. ${ }^{71}$ For molecule VII, the ringcurrent strength susceptibility calculated at the B3LYP level is even three times larger than the one obtained at the MP2 level. According to the MP2 calculations, molecule IV is slightly more antiaromatic than molecule $\mathrm{V}$, whereas the aromaticity order is the same at the B3LYP and MP2 levels for the rest of the studied molecules. Magnetic properties of the antiaromatic porphyrinoids are discussed in more detail elsewhere, ${ }^{71}$ whereas we here focus on the relations between ring-current strength susceptibilities and spectroscopic properties.

\section{Spectroscopic properties}

B3LYP calculations: The vertical excitation energies (VEE), oscillator strengths $\left(f_{e l}\right.$ and $f_{\text {mag }}$ ), electric dipoletransition moments $(\langle 0|\hat{d}| n\rangle)$, and magnetic dipoletransition moments $(\langle 0|\hat{M}| n\rangle)$ calculated at the B3LYP level for the five lowest $\mathrm{S}_{0} \rightarrow S_{n}$ electronic transitions are reported in Table 2. Free-base porphyrin $\left(\mathrm{H}_{2} \mathrm{P}\right)$ and molecules III, VI and VII belong to the $D_{2 h}$ point group. The molecular structures of I, II, IV and V belong to the $C_{2}, C_{s}, C_{s}, D_{4 h}$ point groups, respectively. The relation between the VEEs of the five lowest $S_{0} \rightarrow S_{n}$ transitions and the ring-current strength susceptibility of the porphyrinoid macroring calculated at the B3LYP level is shown in Figure 2.

Molecule I with a net ring-current strength susceptibility of $-1.1 \mathrm{nA} / \mathrm{T}$ is practically nonaromatic, while molecules $\mathrm{VI}$ and VII with ring-current strength susceptibilities of -76.80 $\mathrm{nA} / \mathrm{T}$ and $-145.0 \mathrm{nA} / \mathrm{T}$ at the B3LYP level are strongly antiaromatic according to the ring-current criterion. Antiaromaticity is known to reduce the energy gap between the highest occupied molecular orbital (HOMO) and the lowest unoccupied molecular orbital (LUMO). Antiaromaticity also leads to a smaller VEE of the first excited state for strongly antiaromatic molecules than for molecules sustaining a weaker ring current. ${ }^{85,86}$ Figure 2 shows that the VEE of the $S_{0} \rightarrow S_{1}$ transition of the studied molecules becomes systematically smaller with increasing ring-current strength susceptibility. The VEE of the higher excited states are more or less independent of the size of the ring-current strength susceptibility suggesting that the dominating coefficient in Eq. (2) involves the lowest excited state. Thus, the main contribution to the paratropic contribution of the current density of the antiaromatic porphyrinoids originates from the first excited state. By using Hückel theory, Steiner and Fowler showed that paratropic ring currents characterizing antiaromaticity involves the two electrons of the non-degenerate HOMO, whereas the diatropic ring current of aromatic molecules can be attributed to the electrons of the two nearly degenerate HOMOs. ${ }^{8,87-89}$

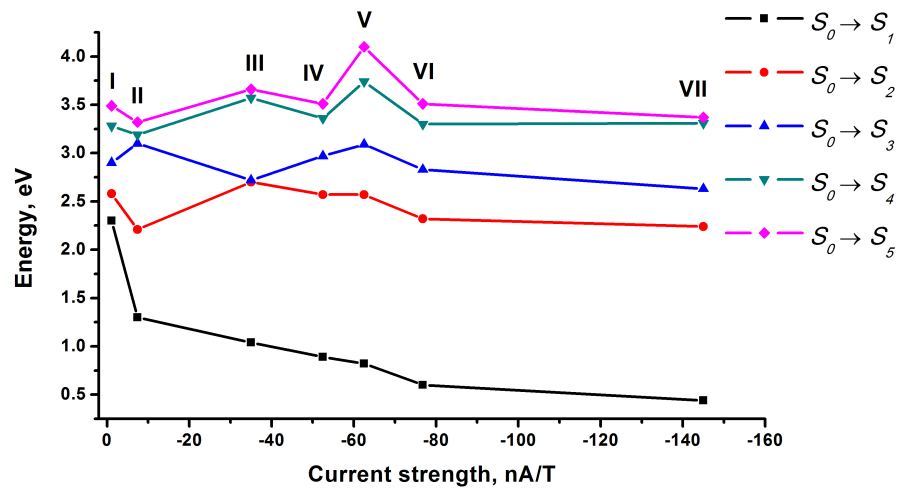

Fig. 2 The vertical excitation energies for the five lowest electronic excited states of compounds I-VII as a function of the ring-current strength susceptibility calculated at the B3LYP level. 
Table 2 The five lowest vertical excitation energies (VEE in $\mathrm{eV})$ and oscillator strengths $\left(f_{e l}\right.$ for electric and $f_{\text {mag }}$ for magnetic dipole transitions) for the studied molecules calculated at the B3LYP/TZVP level using the TDDFT approach. The electric $(\langle 0|\hat{d}| n\rangle)$ and magnetic dipole moments $(\langle 0|\hat{M}| n\rangle)$ (in a.u.) of $\mathrm{S}_{0} \rightarrow \mathrm{S}_{n}$ transitions are also reported.

\begin{tabular}{|c|c|c|c|c|c|c|c|c|c|c|}
\hline & \multicolumn{5}{|c|}{ (I) } & \multicolumn{5}{|c|}{ (II) } \\
\hline States & VEE & $\langle 0|\hat{d}| n\rangle$ & $f_{e l}$ & $\langle 0|\hat{M}| n\rangle$ & $f_{\text {mag }}$ & VEE & $\langle 0|\hat{d}| n\rangle$ & $f_{e l}$ & $\langle 0|\hat{M}| n\rangle$ & $f_{\text {mag }}$ \\
\hline 1 & 2.33 & 0.003 & 0.0001 & 3.59 & 0.73 & 1.31 & 0.79 & 0.02 & 3.88 & 0.48 \\
\hline 2 & 2.58 & 2.00 & 0.25 & 0.00 & 0.00 & 2.21 & 1.18 & 0.07 & 0.37 & 0.01 \\
\hline 3 & 2.90 & 0.00 & 0.00 & 2.81 & 0.56 & 3.10 & 0.87 & 0.06 & 0.86 & 0.06 \\
\hline 4 & 3.28 & 0.00 & 0.00 & 0.44 & 0.02 & 3.19 & 3.15 & 0.78 & 0.50 & 0.02 \\
\hline \multirow[t]{2}{*}{5} & 3.49 & 1.46 & 0.28 & 0.06 & 0.00 & 3.32 & 0.92 & 0.07 & 0.44 & 0.02 \\
\hline & \multicolumn{5}{|c|}{ (III) } & \multicolumn{5}{|c|}{ (IV) } \\
\hline States & VEE & $\overline{\langle 0|\hat{d}| n\rangle}$ & $f_{e l}$ & $\langle 0|\hat{M}| n\rangle$ & $f_{\text {mag }}$ & VEE & $\langle 0|\hat{d}| n\rangle$ & $f_{e l}$ & $\langle 0|\hat{M}| n\rangle$ & $f_{m a g}$ \\
\hline 1 & 1.04 & 0.00 & 0.00 & 4.91 & 0.61 & 0.89 & 0.00 & 0.00 & 4.90 & 0.52 \\
\hline 2 & 2.70 & 0.54 & 0.02 & 0.00 & 0.00 & 2.57 & 0.86 & 0.05 & 0.00 & 0.00 \\
\hline 3 & 2.72 & 0.40 & 0.01 & 0.00 & 0.00 & 2.97 & 0.62 & 0.03 & 0.00 & 0.00 \\
\hline 4 & 3.57 & 3.38 & 0.99 & 0.00 & 0.00 & 3.36 & 0.00 & 0.00 & 0.00 & 0.00 \\
\hline \multirow[t]{2}{*}{5} & 3.66 & 3.81 & 1.30 & 0.00 & 0.00 & 3.51 & 2.24 & 0.43 & 0.00 & 0.00 \\
\hline & \multicolumn{5}{|c|}{$\overline{\overline{(V)}}$} & \multicolumn{5}{|c|}{ 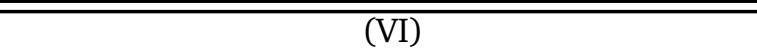 } \\
\hline States & VEE & $\langle 0|\hat{d}| n\rangle$ & $f_{e l}$ & $\langle 0|\hat{M}| n\rangle$ & $f_{\text {mag }}$ & VEE & $\langle 0|\hat{d}| n\rangle$ & $f_{e l}$ & $\langle 0|\hat{M}| n\rangle$ & $f_{\text {mag }}$ \\
\hline 1 & 0.83 & 0.00 & 0.00 & 4.92 & 0.49 & 0.66 & 0.35 & 0.00 & 4.94 & 0.39 \\
\hline 2 & 2.57 & 0.99 & 0.06 & 0.00 & 0.00 & 2.32 & 0.40 & 0.01 & 0.04 & 0.00 \\
\hline 3 & 3.09 & 1.01 & 0.08 & 0.00 & 0.00 & 2.83 & 0.39 & 0.01 & 0.02 & 0.00 \\
\hline 4 & 3.74 & 3.87 & 1.37 & 0.00 & 0.00 & 3.30 & 3.30 & 0.88 & 0.03 & 0.00 \\
\hline \multirow[t]{2}{*}{5} & 4.10 & 3.52 & 1.24 & 0.00 & 0.00 & 3.51 & 2.95 & 0.75 & 0.20 & 0.00 \\
\hline & \multicolumn{5}{|c|}{ (VII) } & & & & & \\
\hline States & VEE & $\langle 0|\hat{d}| n\rangle$ & $f_{e l}$ & $\langle 0|\hat{M}| n\rangle$ & $f_{\text {mag }}$ & & & & & \\
\hline 1 & 0.45 & 0.00 & 0.00 & 5.26 & 0.30 & & & & & \\
\hline 2 & 2.24 & 0.47 & 0.01 & 0.02 & 0.00 & & & & & \\
\hline 3 & 2.63 & 0.26 & 0.00 & 0.02 & 0.00 & & & & & \\
\hline 4 & 3.31 & 2.71 & 0.60 & 0.02 & 0.00 & & & & & \\
\hline 5 & 3.37 & 3.66 & 1.11 & 0.06 & 0.00 & & & & & \\
\hline
\end{tabular}

The energies of the frontier orbitals calculated at the B3LYP level are shown in Figure 3. Since the ringcurrent strength susceptibility increases from molecule I to VII, the graph shows that the HOMO-LUMO gap systematically decreases with increasing paratropic ring-current strength susceptibility. The energy difference between HOMO and HOMO-1 increases from left to right in Figure 3, whereas the orbital energies of LUMO $+1, \mathrm{LUMO}+2$, and HOMO-2 are almost independent of the size of the ring-current strength susceptibility. The magnetic dipole moments of the $\mathrm{S}_{0} \rightarrow S_{n}, n=1,5$ transitions are given in Table 2. The magnetic transition moment to the first excited state is almost constant for molecules III-VII. Molecules I and II have slightly smaller magnetic transition moments than molecules III-VII. The higher-lying excited states of molecules I and II have significant magnetic transition moments, whereas for molecules III-VII they are very small.

The decreasing VEE of the first excited state $\left(\Delta E_{10}\right)$ combined with a large value for the transition matrix element of the $z$ component of the angular momentum operator $\left(\left\langle 1\left|\hat{L}_{z}\right| 0\right\rangle\right)$ leads to a very strong paratropic ring-current strength susceptibilities for molecules III-VII. The $\langle 1|\hat{L}| 0\rangle$ matrix element is completely dominated by the $\left\langle 1\left|\hat{L}_{z}\right| 0\right\rangle$ component, where the $z$ axis is assumed to be perpendicular to the porphyrinoid macroring.

For all molecules except I, II and VI, the electric dipole moment of the $\mathrm{S}_{0} \rightarrow S_{1}$ transition vanishes for symmetry reasons. Thus, for molecules III, IV, V and VII, the first band in the electronic absorption spectrum corresponds to a purely magnetic dipole-allowed transition. The weak $\mathrm{S}_{0} \rightarrow S_{1}$ transition of classic porphyrins like free-base porphyrin is a vibrationally enhanced electric transition due to the Herzberg-Teller effect. ${ }^{62}$ The $\mathrm{S}_{0} \rightarrow S_{1}$ transition of the studied antiaromatic porphyrinoids is magnetic dipoleallowed, which is supported by a recent experimental investigation of spectroscopic properties of antiaromatic expanded porphyrins. ${ }^{39}$

The lowest magnetic dipole-allowed transition from HOMO to LUMO has a weight of 0.7 in the TDDFT calculation of the lowest excited state. For compound I, the 
corresponding transition is from $\mathrm{HOMO}$ to $\mathrm{LUMO}+1$ with weight of 0.7 .

The LUMO of free-base porphyrin $\left(\mathrm{H}_{2} \mathrm{P}\right)$ belonging to the $B_{2 g}$ irreducible representation becomes the HOMO in the formally antiaromatic porphyrinoids. The LUMO +1 of $\mathrm{H}_{2} \mathrm{P}$ belonging to the $B_{3 g}$ irreducible representation is the LUMO for molecules II-VII. For them, the transition from HOMO $\left(B_{2 g}\right)$ to LUMO $\left(B_{3 g}\right)$ is electric dipole forbidden, because the orbitals have the same gerade parity. Instead, the transition is magnetic dipole allowed. Even though molecules II and V have a lower symmetry than $D_{2 h}$, the HOMO and LUMO look like the $B_{2 g}$ and $B_{3 g}$ orbitals of $\mathrm{H}_{2} \mathrm{P}$. Since $D_{2 h}$ is a subgroup of $D_{4 h}$, the HOMO and LUMO of molecule $\mathrm{V}$ can readily be assigned to the $B_{2 g}$ and $B_{3 g}$ irreproducible representations of the $D_{2 h}$ point group.

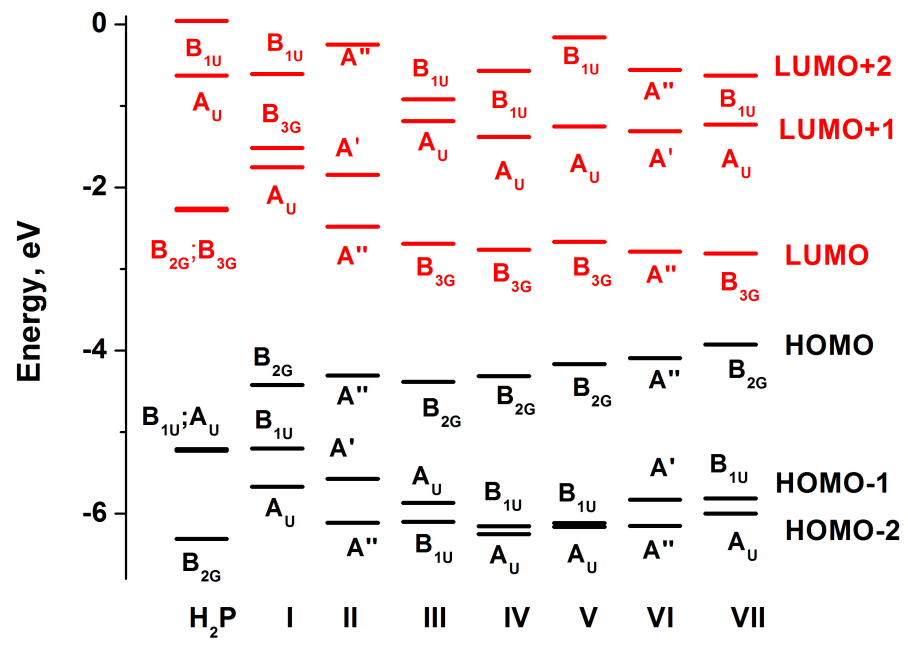

Fig. 3 The energies of HOMO, HOMO- 1 and LUMO, LUMO+1 calculated for compounds I-VII at the B3LYP level. The irreducible representations of the frontier orbitals are also given. The symmetry of the HOMO and LUMO of molecules II, VI, and $V$ are almost the same as for the $B_{2 g}$ and $B_{3 g}$ LUMOs of $\mathrm{H}_{2} \mathrm{P}$.

The LUMO of molecule I is reminescent of the $A_{u} \mathrm{HOMO}$ of $\mathrm{H}_{2} \mathrm{P}$. HOMO and LUMO+1 of molecule I belong to the $B$ irreducible representation implying that the transition is both magnetic dipole allowed with $\langle 0|\hat{M}| 1\rangle \neq 0$ and electric dipole allowed with $\langle 0|\hat{d}| 1\rangle \neq 0$. For molecules II and VI, HOMO and LUMO belong to the $A^{\prime \prime}$ irreducible representation which means that the lowest transition for II and VI is weakly electric dipole allowed, because they do not have any reflection symmetry and therefore lack parity based selection rules. However, for I, II and VI the magnetic transition moment $(\langle 0|\hat{M}| 1\rangle)$ is larger than the electric one $(\langle 0|\hat{d}| 1\rangle)$ due to the electronic nature of these states.

The matrix element of the magnetic dipole transition is rather large for the transition to the first excited state, implying that it is responsible for the strong paratropic ringcurrent strength susceptibility characterizing the antiaromatic nature of the studied porphyrinoids. The HOMOLUMO transition does not contribute to the $\mathrm{S}_{0} \rightarrow S_{n}, n=$ $2-5$ transitions as shown in the ESI $\dagger$.
For molecules II-VII, the electric dipole transition direction of $\mathrm{S}_{0} \rightarrow S_{2}$ and $\mathrm{S}_{0} \rightarrow S_{3}$ are perpendicular to each other in the molecular plane. They correspond to the $Q_{x}$ and $Q_{y}$ bands of classical porphyrins. ${ }^{90,91}$ The individual transition moments are given in the ESI $\dagger$. For the nonaromatic compound I, it was not possible to unambiguously assign the $Q$ bands. However, the $Q_{x}$ and $Q_{y}$ bands are most likely the weak transitions appearing in the typical spectral region for $Q_{x}$ and $Q_{y}$ transitions. All reported transitions are of $\pi$ $\pi^{*}$ character as shown by the plot of the frontier molecular orbitals in the ESI†.

The higher lying $\mathrm{S}_{0} \rightarrow S_{4}$ and $\mathrm{S}_{0} \rightarrow S_{5}$ electronic transitions are assigned to the $B_{x}$ and $B_{y}$ Soret bands for compounds II, III, and V-VII, because they have large electric dipole moments that are in the molecular plane and they are perpendicular to each other. The calculated VEEs are similar to those obtained for classic porphyrins. ${ }^{61,90}$ The $\mathrm{S}_{0} \rightarrow S_{4}$ transition of compounds I and IV is assigned to the $B_{x}$ band. However, since the electric dipole moment of $\mathrm{S}_{0} \rightarrow S_{5}$ for I and IV vanishes, the transition corresponding to the $B_{y}$ band is not among the studied five excited states.

CC2 calculations: The vertical CC2 excitation energies calculated for the five lowest states are reported in Table 3. The CC2 excitation energies are systematically larger than those obtained in the TDDFT calculations using the B3LYP functional. The VEE of the first excited singlet state of the molecule VII with the largest paratropic ringcurrent strength susceptibility is $0.81 \mathrm{eV}$ at the extended multi-configuration quasi-degenerate perturbation theory at second-order (XMC-QDPT2) level. ${ }^{71}$ At the CC2 level, the corresponding VEE is $0.79 \mathrm{eV}$ and the B3LYP calculation yields $0.45 \mathrm{eV}$. The VEE of the first triplet state of molecule VII is $0.52 \mathrm{eV}$ as obtained at the XMC-QDPT2 level of theory. The optical gap calculated at the CC2 level agrees well the one calculated at the XMC-QDPT2 level. The XMC-QDPT2 calculations also show that the ground state of molecule VII is a closed-shell singlet. Thus, the ground state of all the considered molecules are most likely closed-shell singlets.

The $\mathrm{D}_{1}$ diagnostics calculated at the CC2 level are in the range of 0.08 to 0.12 , which is somewhat larger than the usually accepted value. ${ }^{92}$ However, CC2 calculations on DNA bases showed that accurate excitation energies are obtained at the CC2 level when the $\mathrm{D}_{1}$ diagnostic value does not exceed $0.15 .{ }^{93}$ Multi-configuration character as indicated with a large $\mathrm{D}_{1}$ values usually leads to an underestimation of the VEEs calculated at the CC2 level. ${ }^{93}$

A larger energy difference in the denominator of Eq. (2) leads to a smaller contribution to the paramagnetic current density, which explains why the ring-current strength susceptibilities for the strongly antiaromatic molecules are smaller at the MP2 level than obtained in the B3LYP calculations, see Table 1.

The graphs in Figures 2 and 4 show that B3LYP and MP2/CC2 calculations yield the same trends for the exci- 
Table 3 The five lowest vertical excitation energies (VEE in $\mathrm{eV})$ and oscillator strengths $\left(f_{e l}\right.$ for electric and $f_{\text {mag }}$ for magnetic dipole transitions) for the studied molecules calculated at the CC2/TZVP level using the RVS approach. The electric $(\langle 0|\hat{d}| n\rangle)$ and magnetic dipole moments $(\langle 0|\hat{M}| n\rangle)$ (in a.u.) of $\mathrm{S}_{0} \rightarrow \mathrm{S}_{n}$ transitions are also reported.

\begin{tabular}{|c|c|c|c|c|c|c|c|c|c|c|}
\hline & & & (I) & & & & & (II) & & \\
\hline States & VEE & $\langle 0|\hat{d}| n\rangle$ & $f_{e l}$ & $\langle 0|\hat{M}| n\rangle$ & $f_{\text {mag }}$ & VEE & $\langle 0|\hat{d}| n\rangle$ & $f_{e l}$ & $\langle 0|\hat{M}| n\rangle$ & $f_{\text {mag }}$ \\
\hline 1 & 2.88 & 0.01 & 0.0001 & 4.52 & 1.45 & 1.62 & 0.86 & 0.04 & 4.07 & 0.66 \\
\hline 2 & 3.16 & 2.69 & 0.59 & 0.11 & 0.001 & 2.54 & 1.53 & 0.15 & 0.42 & 0.01 \\
\hline 3 & 3.60 & 0.00 & 0.00 & 2.20 & 0.43 & 3.35 & 3.18 & 0.88 & 0.14 & 0.002 \\
\hline 4 & 3.89 & 0.07 & 0.0005 & 0.26 & 0.02 & 3.51 & 0.51 & 0.06 & 0.50 & 0.04 \\
\hline \multirow[t]{2}{*}{5} & 4.04 & 1.32 & 0.17 & 0.03 & 0.0001 & 3.72 & 1.19 & 0.13 & 0.36 & 0.015 \\
\hline & \multicolumn{5}{|c|}{ (III) } & \multicolumn{5}{|c|}{ (IV) } \\
\hline States & VEE & $\langle 0|\hat{d}| n\rangle$ & $f_{e l}$ & $\langle 0|\hat{M}| n\rangle$ & $f_{m a g}$ & VEE & $\langle 0|\hat{d}| n\rangle$ & $f_{e l}$ & $\langle 0|\hat{M}| n\rangle$ & $f_{\text {mag }}$ \\
\hline 1 & 1.56 & 0.00 & 0.00 & 5.20 & 0.61 & 1.25 & 0.00 & 0.00 & 5.17 & 0.82 \\
\hline 2 & 3.25 & 0.76 & 0.05 & 0.00 & 0.00 & 3.08 & 1.37 & 0.14 & 0.00 & 0.00 \\
\hline 3 & 3.38 & 0.65 & 0.04 & 0.00 & 0.00 & 3.37 & 0.94 & 0.07 & 0.00 & 0.00 \\
\hline 4 & 3.95 & 3.60 & 1.26 & 0.00 & 0.00 & 3.81 & 3.18 & 0.95 & 0.00 & 0.00 \\
\hline \multirow[t]{2}{*}{5} & 4.07 & 3.97 & 1.58 & 0.00 & 0.00 & 4.15 & 3.52 & 1.26 & 0.00 & 0.00 \\
\hline & \multicolumn{5}{|c|}{ (V) } & \multicolumn{5}{|c|}{ (VI) } \\
\hline States & VEE & $\langle 0|\hat{d}| n\rangle$ & $f_{e l}$ & $\langle 0|\hat{M}| n\rangle$ & $f_{\text {mag }}$ & VEE & $\langle 0|\hat{d}| n\rangle$ & $f_{e l}$ & $\langle 0|\hat{M}| n\rangle$ & $f_{\text {mag }}$ \\
\hline 1 & 1.26 & 0.00 & 0.00 & 5.20 & 0.84 & 0.99 & 0.41 & 0.004 & 5.04 & 0.62 \\
\hline 2 & 3.20 & 1.81 & 0.26 & 0.00 & 0.00 & 2.80 & 0.31 & 0.007 & 0.05 & 0.0002 \\
\hline 3 & 3.66 & 1.55 & 0.21 & 0.00 & 0.00 & 3.22 & 0.36 & 0.01 & 0.02 & 0.0003 \\
\hline 4 & 4.03 & 3.71 & 1.36 & 0.00 & 0.00 & 3.44 & 3.56 & 1.07 & 0.04 & 0.0002 \\
\hline \multirow[t]{2}{*}{5} & 4.57 & 3.46 & 1.35 & 0.00 & 0.00 & 3.83 & 3.12 & 0.91 & 0.20 & 0.004 \\
\hline & \multicolumn{5}{|c|}{ (VII) } & & & & & \\
\hline States & VEE & $\langle 0|\hat{d}| n\rangle$ & $f_{e l}$ & $\langle 0|\hat{M}| n\rangle$ & $f_{m a g}$ & & & & & \\
\hline 1 & 0.79 & 0.00 & 0.00 & 5.26 & 0.54 & & & & & \\
\hline 2 & 2.75 & 0.49 & 0.01 & 0.016 & 0.00 & & & & & \\
\hline 3 & 3.03 & 0.22 & 0.004 & 0.016 & 0.00 & & & & & \\
\hline 4 & 3.50 & 3.90 & 1.30 & 0.05 & 0.0002 & & & & & \\
\hline 5 & 3.58 & 2.38 & 0.50 & 0.00 & 0.00 & & & & & \\
\hline
\end{tabular}

tation energies as a function of the ring-current strength susceptibility. The main difference is that the excitation energies are shifted to higher values at the CC2 level and that the ring-current strength susceptibilities for the antiaromatic molecules calculated at the MP2 level are systematically smaller than those obtained at the B3LYP level. Molecule V has a slightly smaller ring-current susceptibility than molecule IV at the MP2 level. At the CC2 level, the VEE of the first excited state of molecule V is also slightly smaller than the one for molecule IV. At the B3LYP level, molecule $\mathrm{V}$ has a larger ring-current strength susceptibility and a smaller VEE of the first excited state than for molecule IV.

Comparison of the excitation energies and transition moments calculated at the B3LYP and CC2 levels shows that the first five state of molecules I, III, V, VI and VII have the same character at the two levels of theory. For molecule II, the third and fourth excited states appear in reversed order at the two levels. For molecule IV, the fourth state at the B3LYP level seems to be a spurious state below the $B$ band, whereas at the CC2 level state the fourth and fifth excited states form the $B$ band.

For the antiaromatic molecules according to the ringcurrent strength susceptibility calculated at the MP2 level i.e., molecules III-IV, the first excited state is electric dipole forbidden and magnetic dipole allowed, whereas the second and third excited states can be assigned to the $Q$ band, even though the states have rather large oscillator strengths as compared to the $Q$ bands of free-base porphyrin. The fourth and the fifth excited states of molecules III-VII have large oscillator strengths and can therefore be assigned to the $B$ band.

\section{Summary and conclusions}

Vertical excitation energies (VEE) as well as electric and magnetic transition dipole moments for the five lowest transitions of a set of carbaporphyrins, carbathiaporphyrins and isophlorins have been studied at the time dependent density functional theory (TDDFT) level using the B3LYP functional. The VEEs have also been calculated at the approximate second-order coupled-cluster (CC2) level. 


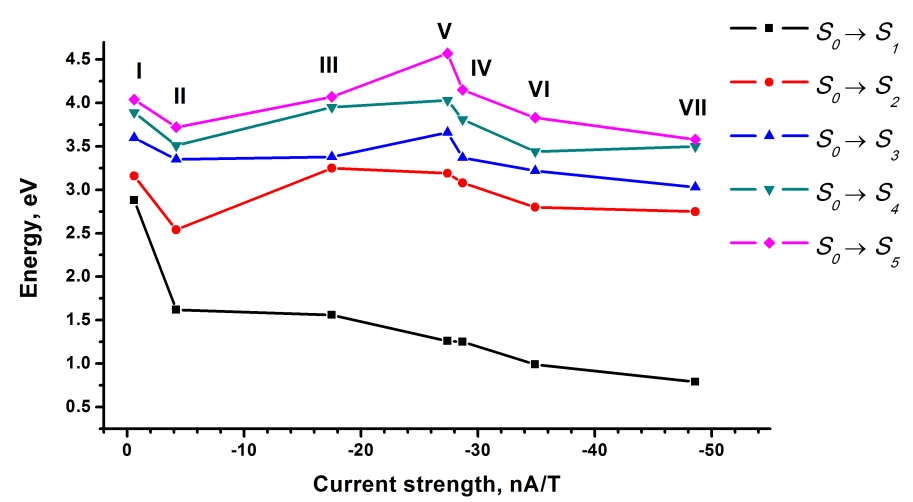

Fig. 4 The CC2 vertical excitation energies for the five lowest electronic excited states of compounds I-VII as a function of the ring-current strength susceptibility calculated at the MP2 level. Note that the order of molecule IV and V are interchanged.

The calculated transition energies and magnetic transition probabilities were compared with the magnetically induced ring-current strength susceptibilities of the porphyrinoid macrorings calculated at the B3LYP, HartreeFock (HF), and second-order Møller-Plesset perturbation theory (MP2) levels of theory.

The calculations show that only the lowest $S_{0} \rightarrow S_{1}$ transition of the studied ones contributes significantly to the magnetically induced ring-current strength susceptibility, because it is the only state for molecules III-VII that has a strong magnetic dipole-allowed transition. Molecules III-VII are antiaromatic according to the ring-current criterion, whereas molecules I and II are almost nonaromatic. The molecules have been labeled such that molecule I is the least antiaromatic molecule and molecule VII has the largest paratropic ring-current strength susceptibility at the B3LYP level. The VEE of the first excited state decreases with increasing size of the magnetically induced paratropic ring-current strength susceptibility, whereas the VEE of the higher-lying states are almost independent of the magnitude of the ring-current strength susceptibility. The magnetic dipole moment of the $S_{0} \rightarrow S_{1}$ transition is almost the same for the antiaromatic molecules III-VII.

For the nonaromatic molecules I and II, the same ringcurrent strength susceptibilities were obtained at the HF and MP2 levels of theory. For molecules III-VI, the MP2 calculations yield ring-current strength susceptibilities that are about a factor of two larger than those obtained at the HF level. B3LYP calculations overestimate the ring-current strength susceptibility of molecules III-VI by about a factor of two as compared to the MP2 values, because the optical gap is systematically smaller at the B3LYP level than obtained in the CC2 calculations. For molecule VII, the ring-current strength susceptibility calculated at the B3LYP level is about three times larger than the one obtained at the MP2 level.

For molecules III, IV, V, and VII the electric dipole transition moment of the $\mathrm{S}_{0} \rightarrow S_{1}$ vanishes due to the symmetry reason. Thus, for these molecules the first band in the elec- tronic absorption spectrum corresponds to a purely magnetic dipole transition. The $\mathrm{S}_{0} \rightarrow S_{1}$ transition seems to be a magnetic dipole-allowed transition only for antiaromatic porphyrinoids, since the weak $\mathrm{S}_{0} \rightarrow S_{1}$ transition of classic porphyrins like free-base porphyrin is an electric dipoleallowed transition. The two extra electrons of the antiaromatic porphyrinoids occupy the LUMO of the classic porphyrins belonging to the $B_{2 g}$ irreducible representation of the $D_{2 h}$ point group. The LUMO of the antiaromatic porphyrinoids belong to the $B_{3 g}$ implying the electric dipole transitions are forbidden, whereas the transition is magnetic dipole-allowed. This transition does not exist in aromatic porphyrinoids, because in for example free-base porphyrin, the HOMO is a $B_{1 u}$ orbital and the LUMO belongs to the $B_{2 g}$ irreducible representation. The HOMO-LUMO transition of free-base porphyrin is an almost symmetryforbidden electric dipole-allowed transition giving rise to the weak $Q$ band in its absorption spectrum.

\section{Acknowledgment}

The Academy of Finland has supported this work through projects 275845 and 297304. We also thank the Norwegian Research Council for support through the CoE Centre for Theoretical and Computational Chemistry (Grant No. 179568/V30 and 231571/F20). We acknowledge computational resources from CSC - IT Center for Science, Finland and from grant No. NN4654K of the Norwegian Supercomputing Program (NOTUR).

\section{References}

1 The Porphyrins, ed. D. Dolphin, Academic Press, London and New York, 1978.

2 E. Hückel, Grundzüge der Theorie ungesättigter und aromatischer Verbindungen, Verlag Chemie, Berlin, 1938.

3 W. von Eggers Doering and F. L. Detert, J. Am. Chem. Soc., 1951, 73, 876-877.

4 M. Gouterman, The Porphyrins Vol. III, Academic Press, London and New York, 1978, p. 1.

5 M. K. Cyrañski, T. M. Krygowski, M. Wisiorowski, N. J. R. van Eikema Hommes and P. von Ragué Schleyer, Angew. Chem. Int. Ed., 1998, 37, 177-180.

6 J. Jusélius and D. Sundholm, Phys. Chem. Chem. Phys., 2000, 2, 2145 2151.

7 J. Jusélius and D. Sundholm, J. Org. Chem., 2000, 65, 5233-5237.

8 E. Steiner and P. W. Fowler, Chem. Phys. Chem., 2002, 3, 114-116.

9 E. Steiner, A. Soncini and P. W. Fowler, Org. Biomol. Chem., 2005, 4, 4053-4059.

10 J.-i. Aihara, E. Kimura and T. M. Krygowski, Bull. Chem. Soc. Jpn, 2008, 81, 826-835.

11 J.-i. Aihara, J. Phys. Chem. A, 2008, 112, 5305-5311.

12 M. Bröring, Angew. Chem. Int. Ed., 2011, 50, 2436-2438.

13 H. Fliegl and D. Sundholm, J. Org. Chem., 2012, 77, 3408-3414.

14 J. I. Wu, I. Fernández and P. von Ragué Schleyer, J. Am. Chem. Soc., $2013,135,315-321$.

15 M. Stepień and L. Latos-Grażyński, Aromaticity in Heterocyclic Compounds, Springer Berlin / Heidelberg, 2009, vol. 19, pp. 83-153.

16 J.-i. Aihara and M. Makino, Org. Biomol. Chem., 2010, 8, 261-266.

17 E. Steiner and P. W. Fowler, Org. Biomol. Chem., 2004, 2, 34-37.

18 J. Ren, F.-Q. Bai and H.-X. Zhang, Int. J. Quant. Chem., 2015, 115, 983-988.

19 P. W. Fowler, M. Lillington and L. P. Olson, Pure Appl. Chem., 2007, 79, 969-979. 
20 P. Fowler, R. Zanasi, B. Cadioli and E. Steiner, Chem. Phys. Letters, 1996, 251, 132-140.

21 R. R. Valiev, H. Fliegl and D. Sundholm, Phys. Chem. Chem. Phys., 2014, 16, 11010-11016.

22 D. Sundholm, H. Fliegl and R. J. Berger, WIREs Comput. Mol. Sci., 2016, 6, 639-678.

23 R. R. Valiev and V. N. Cherepanov, Int. J. Quant. Chem., 2013, 113, 2563-2567.

24 A. Berlicka, P. Dutka, L. Szterenberg and L. Latos-Grażyński, Angew. Chem. Int. Ed., 2014, 53, 4885-4889.

25 T. D. Lash, Eur. J. Org. Chem., 2007, 135, 5461-5481.

26 M. Pawlicki and L. Latos-Grażyński, Chem. Rec., 2006, 6, 64-78.

27 R. R. Valiev, H. Fliegl and D. Sundholm, J. Phys. Chem. A, 2015, 119, 1201-1207.

28 R. R. Valiev, H. Fliegl and D. Sundholm, Phys. Chem. Chem. Phys., 2015, 17, 14215-14222.

29 I. Benkyi, H. Fliegl, R. R. Valiev and D. Sundholm, Phys. Chem. Chem. Phys., 2016, 18, 11932-11941.

30 S. Aronoff and M. Calvin, J. Org. Chem., 1943, 8, 205-223.

31 M. O. Senge, Angew. Chem. Int. Ed., 2011, 50, 4272-4277.

32 K. Berlin, Angew. Chem. Int. Ed., 1996, 35, 1820-1822.

33 T. D. Lash and S. T. Chaney, Chem. Eur. J., 1996, 2, 944-948.

34 J. S. Reddy and V. G. Anand, J. Am. Chem. Soc., 2008, 130, 37183719.

35 B. K. Reddy, A. Basavarajappa, M. D. Ambhore and V. G. Anand, Chem. Rev., 2017, 117, 3420-3443.

36 T. Chatterjee, V. S. Shetti, R. Sharma and M. Ravikanth, Chem. Rev., 2017, 117, 3254-3328.

37 J-Y. Shin, T. Yamada, H. Yoshikawa, K. Awaga, and H. Shinokubo, Angew. Chem. Int. Ed., 2014, 53, 3096-3101.

38 S. Saito and A. Osuka, Angew. Chem. Int. Ed., 2011, 50, 4342-4373.

39 J.-Y. Shin, K. S. Kim, M.-C. Yoon, J. M. Lim, Z. S. Yoon, A. Osuka and D. Kim, Chem. Soc. Rev., 2010, 39, 2751-2767.

40 T. Tanaka and A. Osuka, Chem. Rev., 2017, 117, 2584-2640.

41 S. Shimizu, Chem. Rev., 2017, 117, 2730-2784.

42 C. G. Claessens, D. González-Rodríguez, M. S. Rodríguez-Morgade, A. Medina and T. Torres, Chem. Rev., 2014, 114, 2192-2277.

43 J. L. Sessler, S. J. Weghorn, Y. Hiseada and V. Lynch, Chem. Eur. J., 1995, 1, 56-67.

44 J. L. Sessler, S. J. Weghorn, Y. Hiseada and V. Lynch, J. Am. Chem. Soc., 1990, 112, 2810-2813.

45 L.-L. Lia and E. Wei-Guang Diau, Chem. Soc. Rev., 2013, 42, 291-304.

46 R. Mera-Adasme, W.-H. Xu, D. Sundholm and F. Mendizabal, Phys. Chem. Chem. Phys., 2016, 18, 27877-27884.

47 R. Paolesse, S. Nardis, D. Monti, M. Stefanelli and C. Di Natale, Chem. Rev., 2017, 117, 2517-2583.

48 G. de la Torre, G. Bottari, M. Sekita, A. Hausmann, D. M. Guldi and T. Torres, Chem. Soc. Rev., 2013, 42, 8049-8105.

49 J. Waluk, Chem. Rev., 2017, 117, 2447-2480.

50 J. Mack, Chem. Rev., 2017, 117, 3444-3478.

51 H. M. Goff, Bioinorg. Chem., 1978, 9, 61-79.

52 N. Kobayashi and K. Nakaia, Chem. Comm., 2007, 4077-4092.

53 J. Mack, M. J. Stillman and N. Kobayashi, Coord. Chem. Rev., 2007, 251, 429-453.

54 J. Sebek and P. Bour, J. Phys. Chem. A, 2008, 112, 2920-2929.

55 H. Lu and N. Kobayashi, Chem. Rev., 2016, 116, 6184-6261.

56 J. P. Macquet, M. M. Millard and T. Theophanides, J. Am. Chem. Soc., 1978, 100, 4741-4746.

57 H. Lee, S.-Y. An and C. Minhaeng, J. Phys. Chem. B, 1999, 103, 49924996.

58 J. M. Sung, J. Oh, W.-Y. Cha, W. Kim, J. M. Lim, M.-C. Yoon and D. Kim, Chem. Rev., 2017, 117, 2257-2312.

59 T. V. Esipova, H. J. Rivera-Jacquez, B. Weber, A. E. Masunov, and S. A. Vinogradov, J. Am. Chem. Soc., 2016, 138, 15648-15662.

60 M. Gouterman, J. Mol. Spectrosc., 1961, 6, 138-163.
61 D. Sundholm, Phys. Chem. Chem. Phys., 2000, 2, 2275-2281.

62 F. Santoro, A. Lami, R. Improta, J. Bloino and V. Barone, J. Chem. Phys., 2008, 128, 224311.

63 T. D. Lash, Chem. Rev., 2017, 117, 2313-2446.

64 R. R. Valiev, H. Fliegl and D. Sundholm, J. Phys. Chem. A, 2013, 117, 9062-9068.

65 J. Jusélius, D. Sundholm and J. Gauss, J. Chem. Phys., 2004, 121, 3952-3963.

66 P. W. Atkins and R. S. Friedman, Molecular Quantum Mechanics, Oxford University Press, 1997.

67 A. D. Becke, J. Chem. Phys., 1993, 98, 5648-5652.

68 C. Lee, W. Yang and R. G. Parr, Phys. Rev. B, 1988, 37, 785-789.

69 A. Schäfer, H. Horn and R. Ahlrichs, J. Chem. Phys., 1992, 97, 25712577.

70 F. Weigend and R. Ahlrichs, Phys. Chem. Chem. Phys., 2005, 7, 32973305.

71 R. R. Valiev, H. Fliegl and D. Sundholm, Chem. Comm., 2017, (submitted).

72 M. Casida and M. Huix-Rotllant, Annu. Rev. Phys. Chem., 2012, 63, 287-323.

73 M. J. Frisch, G. W. Trucks, H. B. Schlegel, G. E. Scuseria, M. A. Robb, J. R. Cheeseman, V. G. Zakrzewski, J. A. Montgomery Jr, R. E. Stratmann, J. C. Burant, S. Dapprich, J. M. Millam, A. D. Daniels, K. N. Kudin, M. C. Strain, O. Farkas, J. Tomasi, V. Barone, M. Cossi, R. Cammi, B. Mennucci, C. Pomelli, C. Adamo, S. Clifford, J. Ochterski, G. A. Petersson, P. Y. Ayala, Q. Cui, K. Morokuma, D. K. Malick, A. D. Rabuck, K. Raghavachari, J. B. Foresman, J. Cioslowski, J. V. Ortiz, A. G. Baboul, B. B. Stefanov, G. Liu, A. Liashenko, P. Piskorz, I. Komaromi, R. Gomperts, R. L. Martin, D. J. Fox, T. Keith, M. A. Al-Laham, C. Y. Peng, A. Nanayakkara, C. Gonzalez, M. Challacombe, P. M. W. Gill, B. Johnson, W. Chen, M. W. Wong, J. L. Andres, C. Gonzalez, M. Head-Gordon, E. S. Replogle and J. A. Pople, Gaussian 09, Revision A.02, Gaussian, Inc., Pittsburgh PA, 2016.

74 C. Hättig and A. Köhn, J. Chem. Phys., 2002, 117, 6939-6951.

75 C. Hättig, Adv. Quantum Chem., 2005, 50, 37-60.

76 R. Ahlrichs, M. Bär, M. Häser, H. Horn and C. Kölmel, Chem. Phys. Letters, 1989, 162, 165-169.

77 R. Send, V. R. I. Kaila and D. Sundholm, J. Chem. Phys., 2011, 134, 214114

78 C.-M. Suomivuori, N. O. C. Winter, C. Hättig, D. Sundholm and V. R. I. Kaila, J. Chem. Theory Comput., 2016, 12, 2644-2651.

79 R. Send, C.-M. Suomivuori, V. R. I. Kaila and D. Sundholm, J. Phys. Chem. B, 2015, 119, 2933-2945.

80 R. R. Valiev and E. G. Ermolina and R. T. Kuznetsova and V. N. Cherepanov and D. Sundholm, J. Mol. Model., 2013, 19, 4631-4637.

81 P. Lazzeretti, Prog. Nucl. Magn. Reson. Spectrosc., 2000, 36, 1-88.

82 R. B. Woodward, Angew. Chemie, 1960, 72, 651-662.

83 H. Fliegl, D. Sundholm, S. Taubert, J. Jusélius and W. Klopper, J. Phys. Chem. A, 2009, 113, 8668-8676.

84 H. Fliegl, S. Taubert, O. Lehtonen and D. Sundholm, Phys. Chem. Chem. Phys., 2011, 13, 20500-20518.

85 N. S. Mills, A. Levy and B. F. Plummer, J. Org. Chem., 2004, 69, 66236633.

86 A. Minsky, A. Y. Meyer and M. Rabinovitz, Tetrahedron, 1985, 41 , 785-791.

87 E. Steiner and P. W. Fowler, Phys. Chem. Chem. Phys., 2004, 6, 261.

88 E. Steiner and P. W. Fowler, Chem. Comm., 2001, 2220-2221.

89 E. Steiner and P. W. Fowler, J. Phys. Chem. A, 2001, 105, 9553-9562.

90 R. Bachmann, F. Cerson, C. Cescheidt and E. Vogel, J. Am. Chem. Soc., 1992, 114, 10855-10860.

91 D. Sundholm, Phys. Chem. Chem. Phys., 2003, 5, 4265-4271.

92 C. L. Janssen and I. M. B. Nielsen, Chem. Phys. Letters, 1998, 290 423-430.

93 V. A. Ovchinnikov and D. Sundholm, Phys. Chem. Chem. Phys., 2014 16, 6931-6941. 